\title{
Review Article \\ Conical Intersections Leading to Chemical Reactions in the Gas and Liquid Phases
}

\author{
Yehuda Haas \\ Institute of Chemistry, the Hebrew University of Jerusalem, 91904 Jerusalem, Israel \\ Correspondence should be addressed to Yehuda Haas; yehuda.haas@mail.huji.ac.il
}

Received 2 July 2014; Revised 9 October 2014; Accepted 13 October 2014; Published 16 November 2014

Academic Editor: Rosendo Valero

Copyright (C) 2014 Yehuda Haas. This is an open access article distributed under the Creative Commons Attribution License, which permits unrestricted use, distribution, and reproduction in any medium, provided the original work is properly cited.

\begin{abstract}
The current status of the role of conical intersections (CoIns) in molecular photochemistry is reviewed with a special emphasis on the procedures used to locate them. Due to space limitations, the extensive literature of the subject is given by referring the reader to representative references, whereas the author group's work is described in detail. The basic properties of CoIns are outlined and contrasted with those of transition states in thermal reactions. Location of CoIns using the method of Longuet-Higgins signinverting loops is described in detail. The concept of "anchors"-valence bond structures that represent stable molecules and other stationary points on the potential energy surface-is introduced and its use in constructing loops is described. The authors' work in the field is outlined by discussing some specific examples in detail. Mathematical aspects and details are left out. The main significance of the method is that it explains a large body of photochemical reactions (for instance, ultrafast ones) and is particularly suitable for practicing chemists, using concepts such as reaction coordinates and transition states in the search.
\end{abstract}

\section{Introduction}

Analysis of chemical reactions is usually based on the concept of potential energy surfaces (PESs), which are derived from the Born-Oppenheimer (BO) approximation. Reactions usually start in the ground electronic state, where the reactant is found in a local minimum. Energy must be supplied to the system in order to initiate the reaction and move on to the product. In thermal reactions the energy source is heat. The reaction proceeds along a trajectory that leads adiabatically to a transition state, which is a local maximum along the reaction coordinate (RC). Subsequently, the system goes down to the product. The entire route occurs on one potential surface (PES) only, along a single coordinate; this is thus a one-dimensional (1D) process. The RC is a combination of internal degrees of freedom, usually expressed in normal coordinates. This scenario, introduced in the 1930's, appears to account well for the vast majority of thermal reactions. There are exceptions, of course: some reactions lead to electronically excited states, creating the phenomenon of chemiluminescence [1]. Recently, a new mechanism termed roaming reactions has been introduced [2]. According to this approach, some reactions start out on a certain route (for instance to bond cleavage) but at a certain point begin a roaming motion resulting in a different reaction pattern. Such developments are important but are rather limited compared to the classical transition state theory and are ignored in this work.

In photochemical reactions the energy required to launch the reaction is supplied by light (most frequently UV and visible radiation). Light absorption promotes the molecule to an electronic excited state; thus, the reaction is nonadiabatic, involving more than one PES. Most photochemical reactions end up in the ground state of the products. If, as often happens, the quantum yield of the reaction is smaller than unity part of the electronically excited molecules, return back to the original state of the reactant. Consequently, a mechanism that transfers the molecule from the initial, light induced state to the ground state necessarily exists (possibly via other excited states). As discovered experimentally, this happens even under collision-free conditions.

One ever-present process that accomplishes this task is the radiative pathway, wherein an excited molecule emits a photon (fluorescence, phosphorescence) on returning to the GS. However, many relaxation processes are measured to be much faster than the radiative one; the radiative route 
provides an intrinsic clock to which nonradiative transitions (NRTs) can be compared. The radiative lifetime of a molecule can be derived from the absorption spectrum of the transition, as shown by Einstein's equations. An allowed electronic transition leads to an excited state having a typical radiative lifetime of the order of $10^{-9} \mathrm{~s}$. A fast nonradiative transition is of the order of $10^{-12} \mathrm{~s}$, leading to a fluorescence quantum yield of $10^{-3}$. Faster transitions are referred to as ultrafast and can be probed directly using femtosecond lasers. It turns out that many interesting photochemical reactions proceed at an ultrafast speed, for instance, in biological systems.

Experiment shows that a nonradiative transition between two states becomes faster as they approach each other, an effect expressed by the energy gap law-the probability of crossing between two electronic states, $P$, increases as the energy gap between their potential surfaces decreases $[3,4]$. In many cases the relationship is approximately exponential; that is, $P=\exp (-\Delta E)$ where $\Delta E$ is the energy gap. The maximum rate is attained when the gap vanishes and the two PESs cross. This crossing (a nonadiabatic process) constitutes a violation of the $\mathrm{BO}$ approximation. Thus, in contrast with thermal reactions, the theory of photochemical reactions must seek the conditions under which nonadiabatic processes become efficient.

According to the BO approximation, the total molecular energy (and hence the Hamiltonian) is the sum of an electronic and nuclear terms. The total wave function $\Psi$ may be written as the product of electronic $(\phi)$ and nuclear $(\chi)$ wave functions. In this approximation, the effect of nuclear motion on the electronic wave function is neglected (namely, $\partial \phi / \partial q=0$ for any nuclear coordinate $q$ ). In the Born Oppenheimer (BO) approximation, the electronic PES is the potential under which the nuclei move.

Teller [5] showed that in a polyatomic molecule two coordinates are required to bring about a crossing. When plotted as a function of the two coordinates the PES has the shape of a double cone, the two PESs cross at the apex (where the wavefunction is degenerate); the energy surface near the crossing is called the conical intersection (CoIn). As the crossing is vital in photochemical reactions, it follows that, in contrast with thermal reactions, photochemical ones are $2 \mathrm{D}$ ones.

CoIns were considered for a long time to be rather rare and were largely ignored in the photochemical literature. However, they were of interest to quantum physicists and chemists, who continued to explore their properties. In 1963, Herzberg and Longuet-Higgins [6] in their study of the JahnTeller effect showed that if the electronic wave function was carried through a closed loop around the apex, it changed its sign (underwent a $2 \pi$ phase shift). This came to be known as the sign-change theorem or the geometric phase effect. (Obviously, the nuclear wave-function underwent the same shift, so that the total wave-function did not change.) It was later extended by Longuet-Higgins [7] to any system and numerically demonstrated by using ab-initio electronic structure calculations for the LiNaK system [8]. Mead and Truhlar [9] discussed a related but different approach using vector potentials. Nowadays, there is a consensus that CoIns are quite common and wide-spread. Claims that "In the last two decades, it became clear that state crossings play a central role for internal conversion" are commonly made [10]; see also [11].

The two coordinates $[12,13]$ that lift the degeneracy are defined by two vectors, the derivative coupling one which depends on the coupling between the two states $\left(H_{\text {coupling }} \sim\right.$ $\left.\left\langle\phi_{i}|\partial / \partial q| \phi_{j}\right\rangle\right)$ and the energy difference gradient one, called the tuning vector $\left(H_{\text {tuning }}=\partial\left|E_{i}-E_{j}\right| / \partial q\right)$. The first is nonsymmetric and leads, in a $1 \mathrm{D}$ space, to avoided crossing; the second tunes the energy difference to achieve crossing and is a symmetric one. Their combined effect violates the BO approximation-leading to a nonadiabatic process. The proof that the touching is a CoIn (and not, for instance, a Renner-Teller intersection) is by testing the sign-change upon carrying the electronic wave function in a complete loop around the intersection point. Only a true CoIn will lead to a sign change of the electronic wave function.

In the 1980s interest in CoIns grew, as experiments were extended to the picosecond and subpicosecond time regime. Many ultrafast processes could be accounted for by assuming the involvement of CoIns; in fact, there is still no better explanation. The growing interest in the application of CoIns to photochemical systems led to the development of methods to find them as explained in some representative examples: [13-19]; these methods, based on the concept of vibronic coupling, were crucial for the progress in the field. These efforts were greatly helped by the development of efficient algorithms for calculating wave functions and coupling matrix elements. Computer programs are available to find the minimum energy point in a CoIn (MECI) [2022]; in fact, the CoIn itself is a multidimensional surface so the programs actually find minimum points in a conical intersection crossing seam (MXS). The acronym CoIn refers to this surface, which is in fact a seam and not a single point.

In this review, the focus is on the important special case of a $S_{1} / S_{0}$ intersection, which, like a transition state, is located on both the ground and the excited states' PESs. In distinction with transition states, the CoIn is not a stationary point. The fast passage through a CoIn prevents its direct detection; its presence must, therefore, be experimentally realized by indirect methods only.

An interesting recent development uses some modifications of density functional theory (DFT) for studying CoIns $[23,24]$. In view of the popularity and relatively easy and cheap use of DFT, these developments may become a key factor in the field.

\section{LH Loops and Anchors}

Current quantum chemical calculations are largely performed by advanced methods of the molecular orbital (MO) theory originally developed by Hund, Slater, and Mulliken, such as Hartree-Fock, complete active space (CAS), and multireference configuration interaction (MRCI). In the early days of quantum chemistry, valence bond (VB) theory based on the Heitler-London approximation and developed by Pauling [25] was quite popular. VB is more adapted to conventional chemical concepts, especially bonding and 
resonance structures, than $\mathrm{MO}$ (in which a chemical bond is not defined). Its basic feature is the spin-paired presentation of the chemical bond. VB structures are constructed by considering all possible spin-pairing forms in which two electrons have opposite spins. Unfortunately, VB methods are less suitable to computations than $\mathrm{MO}$ and are rarely used. For a review on VB and MO, [26] may be consulted.

Pross and Shaik [27] offered a simplified presentation of the TS by using the wave functions of the reactant $(R)$ and product $(P)$. At any time during the reaction, the system may be described by a combination of the two:

$$
|R\rangle(t)=c_{R}(t)|R\rangle+c_{P}(t)|P\rangle,
$$

where $c_{R}(t=0)=1, c_{P}(t=0)=0, c_{R}(t=\infty)=0$, and $c_{P}(t=\infty)=1$.

We define an anchor as a stationary point on the PES having a specific spin-pairing scheme, independent of the nuclear configuration. An anchor may consist of a single or a group of $\mathrm{VB}$ structures. If a reaction is elementary, there is only a single transition state between the reactant $R$ and product $P$. In the transition state region, a spin-pairing change must take place. At this nuclear configuration, the electronic wave function may be written as

$$
|\mathrm{TS}\rangle_{\mathrm{el}}=k_{R}|R\rangle_{\mathrm{el}}+k_{P}|P\rangle_{\mathrm{el}} .
$$

If the sign of $k_{R}$ is equal to that of $k_{P}$, the TS is the inphase combination of $|R\rangle$ and $|P\rangle$; if the signs are different, TS is their out-of-phase combination (the $k$ 's are assumed positive). The reactions are named p-type and i-type, if the TS is the in-phase (sign-preserving) or out-of-phase (inverting) combinations, respectively. The opposite combination is an electronically excited state. Using the determinant form of the electronic wave functions for Hückel-type reactions, which are the most ordinary ones (others are Möbius ones (the concept of Hückel and Möbius type reactions is clearly presented in $[28,29])$. For a generalization of Heilbronner's derivation for Möbius systems bearing one half twist to those bearing $n$ half twists, see [30]), it is readily shown that in i-type reactions an even number of electron pairs are exchanged, whereas in an in-phase reactions, an odd number of electron pairs are exchanged $[31,32]$. Thus, repairing of 4 , $8, \ldots$ pairs leads to i-type reactions, and repairing of $2,6, \ldots$ pairs leads to a p-type reactions.

An anchor, as defined above, contains stable molecules, conformers, all pairs of radicals and biradicals formed by a simple bond fission in which no spin re-pairing took place.

\subsection{The Sign-Change Rule and the Construction of Loops.} According to the sign-change theorem, when a wavefunction of the given electronic state in the $\mathrm{BO}$ approximation is adiabatically transported round a closed path encircling a conical intersection, the wave-function changes its sign. A number of approaches for locating conical intersections based on the sign-change theorem are available, among them the recognition that the sign-inverting method is a variant of Berry's phase [33-36]. Generalizing on the $\mathrm{H} 3$ system $[6,7]$, a loop is constructed by using a sequence of two or three elementary reactions (in both, three or more VB structures are required). The reactions comprising the loop must be elementary, having a single TS. According to the uniqueness theorem [37] if a loop formed by two or three elementary reactions is sign-inverting, it encircles one and only one conical intersection.

The following terminology is used: each reaction in the loop is termed a leg, which is either p-type or i-type. A threelegged loop is sign inverting if it is an $\mathrm{i}^{3}$ or ip $\mathrm{p}^{2}$ loop, and signpreserving otherwise. A two-legged loop is sign inverting if the two TSs differ (one is p-type, the other i-type) and signpreserving otherwise.

Crossings between more than two states [38-40] have been discussed in the literature but are out of the scope of this paper.

\section{Goal and Distinction of the $\mathbf{L H}$ Loop Method}

There are numerous excellent procedures for finding and optimizing CoIns. One might wonder what new message is provided by the present method; it does not endeavor to present a better or more elegant optimization procedure. Its main distinction is in the beginning stage of the search. Other programs use a guess, based on intuition and chemical properties, and invest most of the effort in the optimization procedure. The need for optimization is not immediately obvious. The search for it implicitly assumes that the system will tend to reach the minimum energy of the CoIn (a multidimensional surface) prior to the actual crossing. This is not necessarily the case and is yet to be demonstrated. More important, the (educated) guess may be wrong and lead to nonphysical species. In the proposed method, the structures of reactant and product are essential for constructing the loop, so that no guess is required. At the same time, if the loop is indeed sign-inverting, as can be verified without the need of a calculation, a conical intersection is bound to be found within it. Moreover, the method is particularly useful in the case of large molecules, when several reaction paths are possible. In it, the desired reaction is chosen and a CoIn is sought specifically for that reaction.

\section{Examples}

Many systems were analyzed using the elementary reaction scheme. These include the butadiene-cyclobutene one [41, 42], cis-trans isomerization of ethylene and polyenes [43-45], the $\mathrm{E}-\mathrm{Z}$ isomerization of the formaldiminium cation [37], the allyl radical-cyclopropyl radical ring closure reaction [44], and most recently styrene, stilbene, and the protonated Schiff base, PSB3 [24].

The basic concepts and their advantages are exemplified by a discussion of benzene photochemistry. Benzvalene is one of the products of the $S_{1}$ photolysis of benzene [46]; the sole observable product of the thermal isomerization of benzvalene is benzene [47]. Haas and Zilberg [44], in their study of the photochemical reaction, chose benzene (reactant) and benzvalene (BZ1, product) as two of the anchors. The third 
anchor was another benzvalene isomer, BZ2. The reasoning was that the BZ1 to BZ2 conversion is antisymmetric, and the two BZ to benzene reactions are of the same symmetry, so that the loop is sign-inverting and a conical intersection must be present.

Vanni et al. [48] discussed the same reaction. They offered a reformulation of the elementary reaction loop method and pointed out its connection with the concepts of coupling and tuning vectors used in the CoIn literature. They also criticized the work on the benzvalene to benzene reaction. Two BZ isomers were used as anchors, although a physical argument justifying this choice was not given.

It was pointed out that the selection of benzene as a third anchor cannot be correct because once two anchors (i.e., VB structures) are chosen all possible remaining anchors are unambiguously determined as combinations of the two, but no such combination can be found to represent benzene. A biradical structure, termed prebenzvalene (Figure 14 in [48]), was suggested as the third anchor. In an earlier paper [49], this structure was found computationally (CASSCF/4-31G) to be a local minimum on the $S_{0}$ surface having a barrier of $1.3 \mathrm{kcal} / \mathrm{mol}$ to form benzene and $0.1 \mathrm{kcal} / \mathrm{mol}$ to form benzvalene. These tiny activation energies were considered by others to be insufficient evidence for a real minimum [50].

Since Haas and Zilberg actually found a sign inverting loop and a CoIn in their analysis, an explanation is called for. The prebenzvalene entity is not an experimentally observable species; may it be replaced by benzene? These two molecules are each represented by two VB spin-pairing schemes. Inspection shows that one of the benzene $\mathrm{VB}$ structures (one Kekule structure) is transformed to one VB prebenzvalene structure by a four electron re-pairing scheme and the other by another four-electron scheme. This may be interpreted to represent a sign-inverting elementary reaction, as suggested by Palmer's results [49]. This reaction is quantitative-no other product is formed. Thus, although formally benzene cannot be the third anchor, for all practical purposes, it is. The upshot of this analysis is that both methods lead to the same result. Therefore, in establishing the connection to experiment, whether prebenzvalene is a minimum or not is inconsequential; benzene can be the third anchor in the benzvalene-benzene reaction.

\section{Solvent Effect and Two-Legged Loops}

Most of the theoretical work on CoIns dealt with isolated molecules; in contrast most photochemical experiments are conducted in the bulk. In this section the effect of external fields and of solvents on the properties of CoIns is discussed. The topic is introduced using an example of two-legged loops, a circumstance that is often encountered in the field.

Consider a reactant and product whose ground states have an appreciable polar character.

The VB presentations may be written:

$$
\begin{aligned}
& |R\rangle=\left|R_{\text {cov } 1}\right\rangle+\left|R_{\text {ion }}\right\rangle, \\
& |P\rangle=\left|P_{\text {cov } 2}\right\rangle+\left|P_{\text {ion }}\right\rangle,
\end{aligned}
$$

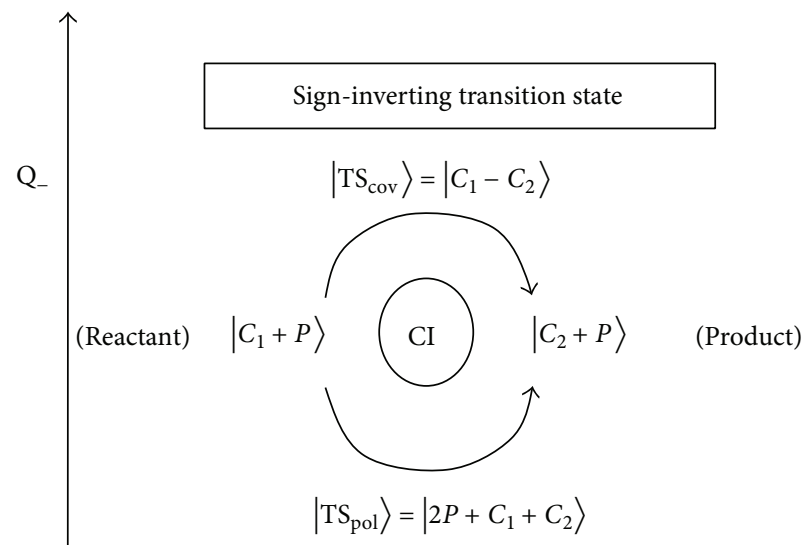

Sign-preserving transition state

$\mathrm{Q}_{+}$

FIGURE 1: An example of two-legged sign-inverting domain, adapted from [37]. CI is a conical intersection; TS is a transition state.

(numerical coefficients and normalization are suppressed, to simplify notation).

An example is an olefin carrying electron donor and electron acceptor groups at opposite sides of the $\mathrm{C}=\mathrm{C}$ double bond. The reaction is the cis-trans isomerization around the double bond. For simplicity the polar part is identical for the two molecules. Two reaction coordinates, having two TSs, can be constructed. The EWF of the transition states are the out-of-phase and in-phase combinations of the EWFs of $R$ and $P$ :

$$
\begin{gathered}
\left|\mathrm{TS}_{\mathrm{cov}}\right\rangle=|R\rangle-|P\rangle=\left|R_{\mathrm{cov} 1}\right\rangle-\left|R_{\mathrm{cov} 2}\right\rangle \\
\text { Sign-inverting, } \\
\left|\mathrm{TS}_{\mathrm{ion}}\right\rangle=|R\rangle+|P\rangle=2\left|R_{\mathrm{ion}}\right\rangle+\left|R_{\mathrm{cov} 1}\right\rangle+\left|R_{\mathrm{cov} 2}\right\rangle
\end{gathered}
$$

Sign-preserving.

A two legged loop can be constructed as shown in Figure 1. Due to the symmetry properties of the two TSs, it is a signinverting loop and therefore contains a CoIn.

Examples for these reaction types abound, for instance, the photoisomerization of the formaldiminium cation and of many Schiff bases, important in the vision mechanism. Here it is demonstrated for the cis-trans isomerization of 1-Butyl4-(1H-inden-1-ylidene)-1,4-dihydropyridine [51, 52] (BIDP, Scheme 1, III). This molecule is easier to handle experimentally than 4-cyclopentadienyl-1,4-dihydropyridine, (CPDHP) that was used in the calculations. The structures of the biradical and ionic transition states of CPDHP are shown in Scheme 2.

The ionic form is stabilized in this case by aromatization. The isomerization around the CC double bond can proceed along two reaction coordinates: the usual covalent one with a biradical TS (BRTS) and another one having ionic TS (ZWTS). The reaction coordinate connecting the reactant 
<smiles>C1=CC(=C2C=CNC=C2)C=C1</smiles><smiles>[R]C1=CC(=C2C=Cc3ccccc32)C=CN1[R1]</smiles>

(I) $\mathrm{R}_{1}=\mathrm{R}_{2}=\mathrm{H}$

(II) $\mathrm{R}_{1}=n-\mathrm{Bu}, \mathrm{R}_{2}=\mathrm{H}$

(III) $\mathrm{R}_{1}=n-\mathrm{Bu}, \mathrm{R}_{2}=\mathrm{CH}_{3}$

(a)<smiles>CC1=CC(=C2C=Cc3ccccc32)C=CN1C</smiles>

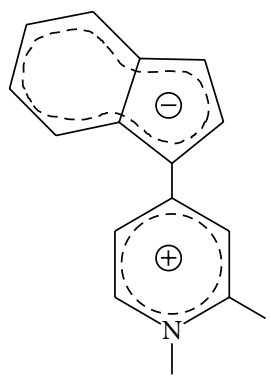

(b)

Scheme 1: (a) The molecular structure of CPDHP (left) and the title compounds (right); II is BIDP and III is BIMDP; (b) the covalent and zwitterion VB structures of I that contribute to the electronic wave-function of the compound.
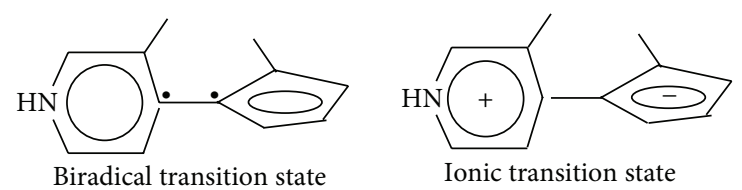

Scheme 2: The structures of the biradical and ionic transition states of CPDHP.

with the product $\left(\mathrm{Q}_{+}\right)$is mainly torsion, whereas the coordinate connecting the two TSs $\left(\mathrm{Q}_{-}\right)$is mainly $\mathrm{C}=\mathrm{C}$ bond stretch and aromatization. A CAS calculation confirmed the presence of a CoIn in the gas phase. The calculation showed that the ground state of CPDHP has a rather small dipole moment in the gas phase $(4.3 \mathrm{D})$, whereas the ZWTS dipole moment $(16.3 \mathrm{D})$ is very large. The BRTS has a dipole moment of only $2.2 \mathrm{D}$; thus, the BRTS has an essentially pure biradical nature and the ZWTS a purely ionic (charge transfer) character. In the gas phase the ZWTS lies at a higher energy than the BRTS (2.05 versus $1.95 \mathrm{eV}$ above the GS). The solvent effect, approximated using the Kirkwood and Onsager model, was calculated to be remarkable. The energy of the BRTS was practically unchanged upon increasing the polarity of the solvent, whereas that of the ZWTS was considerably reduced, becoming $0.96 \mathrm{eV}$ in acetonitrile. The BRTSin this solvent lies in fact on the excited state, and the CoIn becomes an avoided crossing. These results are depicted pictorially in Figure 2, taken from [53].

Further support came from ultrafast measurements of the lifetime of MRCN in different solvents. The two major VB structures of MRCN are shown in Scheme 3: An ionic one (bottom), and a covalent one (top). The lifetime of the excited state is about $300-400 \mathrm{fs}$ in toluene and $900-1000 \mathrm{fs}$ in $\mathrm{MeCN}$, in agreement with the trend predicted by theoretical predictions [54].

\section{Future Research Directions}

The reaction coordinate approach is uniquely suited for studying complex photochemical transformations. In particular it could be used in systems exhibiting several reaction pathways. Prime examples are photobiology, green chemistry, and development of new materials such as molecular switches.

An important and still highly controversial example is the origin of the photostability of DNA and DNA bases, which is believed to have played crucial role in the appearance of life. Recent experiments support a direct transition from the excited state of these molecules to the ground state, circumventing any chemical change in spite of the high energy absorbed (4-6 eV) [55-57]. Most proposed mechanisms for the individual bases and their protonated derivatives involve a CoIn, but its nature is still being debated [58-60]. Ring puckering and frustrated $\mathrm{H}$-atom ejection are among the proposed routes for single bases. Mechanisms suggested include among others deactivation via proton transfer, which is mediated by an excited singlet state of charge-transfer character or vibronic coupling between different pp states. A careful analysis helped by the reaction coordinate method may point out all possible routes, and the competition between them. A more challenging issue is the case of DNA itself. Many chemical processes are possible, especially considering the initial high energy content after photoexcitation. Ground state reactions such as isomerization, cyclobutane formation, and hydrogen atom transfer must be considered and are shown to be less efficient than direct $S_{1}-S_{0}$ transition. The latter necessarily involves a large amplitude motion which might appear as a relatively slow process. The experimental evidence indicates that it is in fact ultrafast. An extensive study of the multidimensional potential energy is probably required.

Another field is the synthesis of novel materials. Considerable efforts are invested currently in environment friendly reactions ("green chemistry"). In this case a central challenge is to replace harmful reactants or solvents with environmentally friendly ones, avoiding toxic, flammable, and/or long-term hazardous products and by-products. Solvents are believed to play a central role; thus, for instance, replacing organic solvents with water could revolutionize the chemical industry. Although some progress has been reported, the much work remains to be accomplished. Photochemistry offers many opportunities that have been so far only superficially considered in the field. However, even solution-free reactions present a challenge. An example is the preparation of fire-works and high energy density materials (HEDMs). 

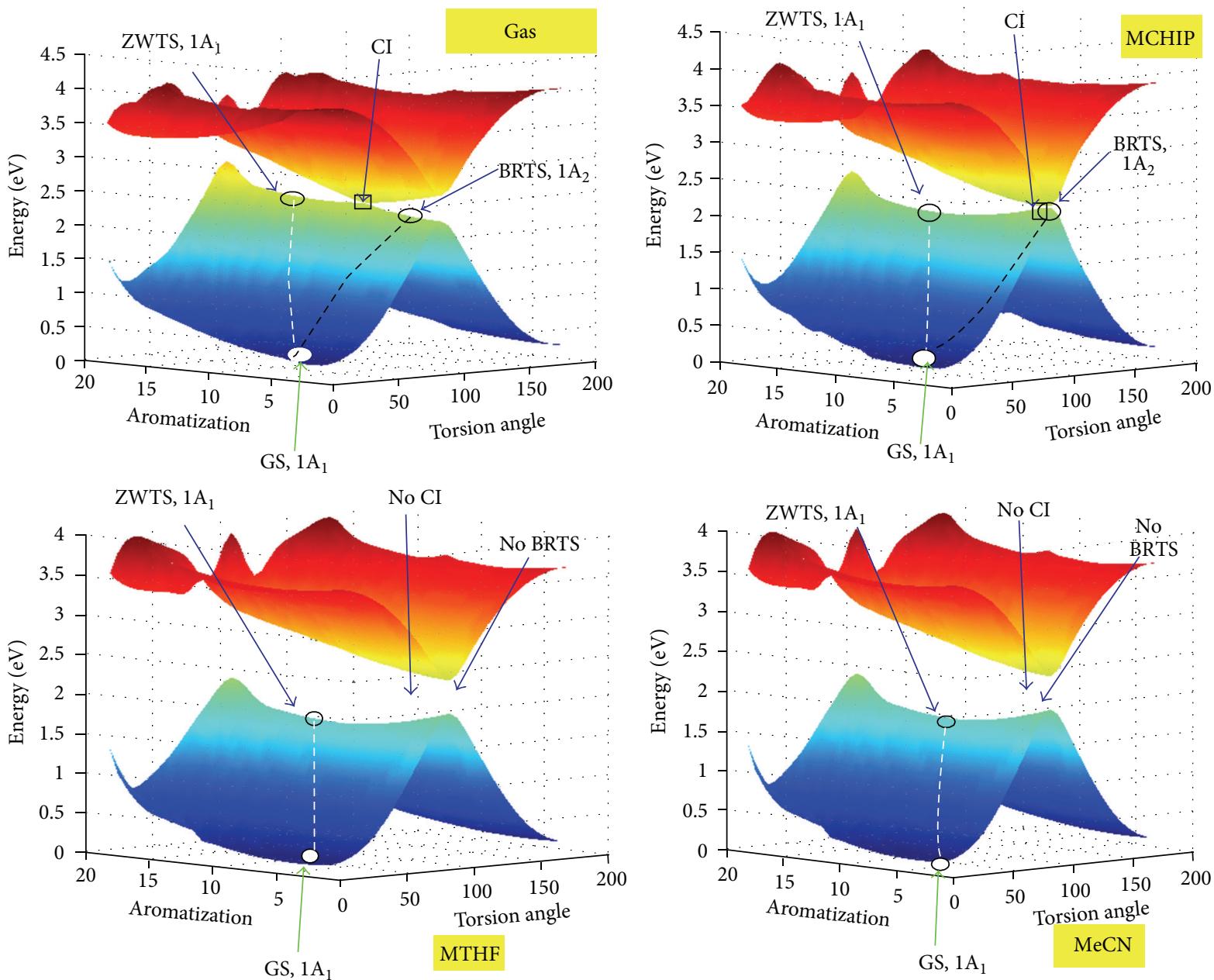

Figure 2: The calculated potential energy surface CPDHP in the gas phase and some solvents in the vicinity of the conical intersection. The positions of the ground state (planar) and two transition states (perpendicular) both having $C_{2 V}$ symmetry are marked in the figure along with that of the CoIn. The approximate trajectories of the two possible thermal reactions are schematically indicated by the dashed curves. In the more polar solvents (MTHF and MeCN) the BR structure lies on $S_{1}$; there is no biradical transition state and the CoIn vanishes [53].
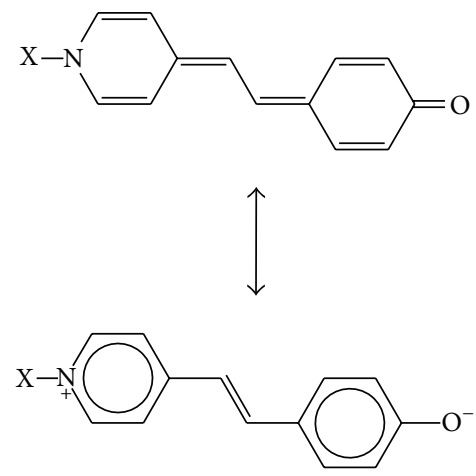

SCHEME 3: The two major VB structures of MRCN.

Effort to produce materials that will replace currently widely used chlorine containing compounds and decompose only (or mainly) to yield $\mathrm{N}_{2}$ gas is underway $[61,62]$.
The preparation of all-nitrogen compounds is of the prime interest, practically and also theoretically. For instance, the cyclo- $\mathrm{N}_{5}{ }^{-}$anion has been prepared in the gas phase $[63,64]$ but not in solution. A photochemical mechanism involving a CoIn [65] has been proposed. A reaction in which an aryl pentazole is the starting material is of interest. The desired product (cyclo- $\mathrm{N}_{5}{ }^{-}$) can be prepared by breaking the $\mathrm{CN}$ bond between the phenyl and the cyclopentazole ring in aryl pentazoles (see Scheme 4 for the structure of an often used example).

However, a reaction having much lower activation energy, extrusion of $\mathrm{N}_{2}$ to form the corresponding azide, is heavily favored thermally. A photochemical process circumventing the ground state reaction to form the less likely product is possible theoretically (resembling, in principle, the ultrafast route in the DNA bases). The reaction coordinate method, being readily adaptable to introduce solvent effects, can help in the search for conditions favoring the thermally less probable route. 


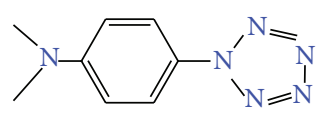

Scheme 4: The structure of an aryl pentazole (para-dimethylamino phenyl pentazol).

\section{Molecular Switches}

Photochemical molecular switches are of great interest as alternatives to the present silicon-based technology. Devices based on reactions such as isomerization around an $\mathrm{N}=\mathrm{N}$ double bond [66, 67] and ring opening [68] are actively being considered as they offer a substantial structural change at an ultrafast rate. Syn-anti rotational isomerism around an $\mathrm{N}=\mathrm{N}$ bond and cyclohexadiene ring opening are only two examples that have been analyzed as passing through a conical intersection. For use in a real device, the molecules must be embedded in a solid matrix, organic or inorganic. The effects of such environments on photochemical reactions have not yet received extensive analysis, although some promising studies have been reported $[69,70]$. This may be one of the important future developments of the field. It is very likely that treating the matrix as a cage will not correctly predict their effect. Rather, changes in electric fields, hydrogen bonding, polarization changes, and other parameters may be used to optimize the desired reaction.

\section{Summary and Conclusions}

The principal motivation of this study is the elucidation of photochemical reaction mechanisms. As the theory of thermal reactions is well-developed, an attractive option is the development of a model on equal (or similar) footing for photochemical ones. Thermal reactions are analyzed as $1 \mathrm{D}$ processes, wherein the concept of transition state plays a major role. For photochemical reactions, two dimensions are needed and the transition between 2 electronic states via a conical intersection is a crucial step. Restricting the scope to the special but important case of reactions involving only the $S_{1}$ and $S_{0}$ states, a method for locating CoIns is introduced. Some CoIn traits resemble those of TSs-both are transient species through which the system must pass during a reaction and their role can be assessed only indirectly. The transit thru both is very rapid-the system never stays in them for a measurable time; both can lead to either the product or back to the reactant. Yet, a CoIn is utterly different from a TS: in addition to requiring two dimensions for its definition, it is a $n-2$ multidimensional surface ( $n$ is the number of nuclear degrees of freedom) and not a point on the PES; no location on this surface is a stationary point (i.e., $\partial E / \partial q \neq 0$ for any $q$ ); although it is part of the $S_{0}$ PES, it is normally not involved in thermal reactions. One might hope that the CoIn concept could provide a common means to analyze many diverse reaction schemes. A desirable (and possibly natural) tactic is to base the analysis on well-founded chemical concepts, such as the ones used in the analysis of thermal reactions. A central concept is that of a chemical bond based on the spin-pairing of two electrons.
This work is a preliminary step in that direction. It shows that a $S_{1} / S_{0}$ CoIn can in principle be located using ground state features only. The actual application uses VB methodology, as it provides a simple means to represent bond breaking and forming. Molecules are represented by anchors, which are VB structures or combination of them, enabling the construction of LH loops. The recent application of $\mathrm{VB}$ theory to the properties of transition states makes this choice a natural one $[71,72]$. Nonetheless, computational implementation is usually by the CI-MO method. The sign variation of the electronic wave-function on traversing the loop signals the presence of a CoIn.

Naturally, problems arise. Some are due to the lack of an exact mathematical description of basic concepts: for instance even the exact definition of a molecule is sometimes arbitrary. The method uses spin-pairing as the practical means for presenting their wave-function. The sign convention of a reaction in which more than one $\mathrm{VB}$ structure is required is not always easy to establish. In particular, participation of several resonance structures may make the sign assignment inconclusive.

A basic impediment encountered in searching for avoided crossings and CoIns has been summarized by Worth and Cederbaum [73] “The complete evaluation of potential energy surfaces is an impossible task for systems comprising more than a few atoms." Thus, some simplifying assumptions have to be made. These assumptions are embodied, for instance, in the approximation made in the initial steps of the search for the CoIn.

The rationale for using reaction coordinates of elementary reactions (RCER) adopted in the present case is to minimize intuitive guess in the initial location of the CoIn. Other methods start at the geometry of a single point (for instance, the minimum energy on the GS surface) and assume that a CoIn exists in its vicinity [74]. The actual search is often initiated on the excited state at this geometry [49]. In the RCER method, it is assumed that the reactant and product VB wave-functions are known, and the third anchor is not independent on the other two-it is some combination of the VB structures that form them. However, elementary reactions can lead instantaneously from the third anchor to a more stable different chemical species (see the discussion of the benzvalene-benzene reaction). Thus, all of stable chemical species involved in the reaction can be observed experimentally. The method is uniquely appropriate for large polyatomic systems, in which several degeneracies are common.

\section{Conflict of Interests}

The author declares that there is no conflict of interests regarding the publication of this paper.

\section{Acknowledgments}

This study was supported by the Deutsche Forschungsgemeinschaft in the framework of Project no. MA 515/22-2. The author is deeply indebted to his coworkers and students, who 
made this work possible: S. Zilberg, B. Dick, J. Manz, W. Fuss, O. Deeb, S. Al-Falah, L. Belau, S. Cogan, X. F. Xu, B. Bazanov, A. Kahan, and U. Geiger.

\section{References}

[1] N. J. Turro, P. Lechtken, N. E. Schore, G. Schuster, H. C. Steinmetzer, and A. Yekta, "Tetramethyl-1,2-dioxetane. Experiments in chemiexcitation, chemiluminescence, photochemistry, chemical dynamics, and spectroscopy," Accounts of Chemical Research, vol. 7, no. 4, pp. 97-105, 1974.

[2] J. M. Bowman and A. G. Suits, "Roaming reactions: the third way," Physics Today, vol. 64, pp. 33-37, 2011.

[3] G. W. Robinson and R. P. Frosch, "Electronic excitation transfer and relaxation," Journal of Chemical Physics, vol. 38, pp. 118-120, 1963.

[4] K. Freed and J. Jortner, "Multiphonon processes in the nonradiative decay of large molecules," The Journal of Chemical Physics, vol. 52, pp. 6272-6291, 1970.

[5] E. Teller, "The crossing of potential surfaces," The Journal of Physical Chemistry, vol. 41, no. 1, pp. 109-116, 1937.

[6] G. Herzberg and H. C. Longuet-Higgins, "Intersection of potential energy surfaces in polyatomic molecules," Discussions of The Faraday Society, vol. 35, pp. 77-82, 1963.

[7] H. C. Longuet-Higgins, "The intersection of potential energy surfaces of polyatomic molecules," Proceedings of the Royal Society of London A, vol. 344, pp. 147-156, 1975.

[8] A. J. C. Varandas, J. Tennison, and J. N. Murrell, "Chercher le croisement," Chemical Physics Letters, vol. 61, pp. 431-434, 1979.

[9] C. A. Mead and D. G. Truhlar, "On the determination of Born-Oppenheimer nuclear motion wave functions including complications due to conical intersections and identical nuclei," The Journal of Chemical Physics, vol. 70, pp. 2284-2296, 1979.

[10] M. Barbatti, "Photorelaxation induced by water-chromophore electron transfer," Journal of the American Chemical Society, vol. 136, no. 29, pp. 10246-10249, 2014.

[11] D. G. Truhlar and C. A. Mead, "The relative likelihood of encountering conical intersections and avoided intersections on the potential energy surfaces of polyatomic molecules," Physical Review A, vol. 68, no. 3, Article ID 032501, pp. 32501-2, 2003.

[12] W. Domcke, D. R. Yarkony, and H. Köppel, Eds., Conical Intersections. Theory, Computation, and Experiment, vol. 17 of Advanced Series in Physical Chemistry, World Scientific, Singapore, 2011.

[13] F. Bernardi, M. Olivucci, and M. A. Robb, "Potential energy surface crossings in organic photochemistry," Chemical Society Reviews, vol. 25, no. 5, pp. 321-328, 1996.

[14] D. R. Yarkony, "Conical intersections: diabolical and often misunderstood," Accounts of Chemical Research, vol. 31, no. 8, pp. 511-518, 1998.

[15] M. Ben-Nun, J. Quenneville, and T. J. Martinez, "Ab initio multiple spawning: photochemistry from first principles quantum molecular dynamics," The Journal of Physical Chemistry A, vol. 104, no. 22, pp. 5161-5175, 2000.

[16] M. A. Robb and M. Olivucci, "Photochemical processes: potential energy surface topology and rationalization using VB arguments," Journal of Photochemistry and Photobiology A: Chemistry, vol. 144, no. 2-3, pp. 237-243, 2001.
[17] A. L. Sobolewski and W. Domcke, "Conical intersections induced by repulsive ${ }^{1} \pi \sigma^{*}$ states in planar organic molecules: malonaldehyde, pyrrole and chlorobenzene as photochemical model systems," Chemical Physics, vol. 259, no. 2-3, pp. 181-191, 2000 .

[18] M. G. D. Nix, A. L. Devine, R. N. Dixon, and M. N. R. Ashfold, "Observation of geometric phase effect induced photodissociation dynamics in phenol," Chemical Physics Letters, vol. 463, no. 4-6, pp. 305-308, 2008.

[19] W. Domcke and G. Stock, "Theory of ultrafast nonadiabatic excited-state processes and their spectroscopic detection in real time," in Advances in Chemical Physics, vol. 100, pp. 1-169, John Wiley \& Sons, New York, NY, USA, 1997.

[20] M. J. Bearpark, A. Michael, M. A. Robb, and H. B. Schlegel, "A direct method for the location of the lowest energy point on a potential surface crossing," Chemical Physics Letters, vol. 223, no. 3, pp. 269-274, 1994.

[21] H. Lischka, M. Dallos, P. G. Szalay, D. R. Yarkony, and R. Shepard, "Analytic evaluation of nonadiabatic coupling terms at the MR-CI level. I. Formalism," The Journal of Chemical Physics, vol. 120, no. 16, pp. 7322-7329, 2004.

[22] M. Dallos, H. Lischka, R. Shepard, D. R. Yarkony, and P. G. Szalay, "Analytic evaluation of nonadiabatic coupling terms at the MR-CI level. II. Minima on the crossing seam: formaldehyde and the photodimerization of ethylene," The Journal of Chemical Physics, vol. 120, no. 16, pp. 7330-7339, 2004.

[23] N. Minezawa and M. S. Gordon, "Optimizing conical intersections of solvated molecules: the combined spin-flip density functional theory/effective fragment potential method," The Journal of Chemical Physics, vol. 137, no. 3, Article ID 034116, 2012.

[24] M. Filatov, "Assessment of density functional methods for obtaining geometries at conical intersections in organic molecules," Journal of Chemical Theory and Computation, vol. 9, no. 10, pp. 4526-4541, 2013.

[25] L. Pauling, The Nature of the Chemical Bond and the Structure of Molecules and Crystals, Cornell University Press, 1939.

[26] M. W. Schmidt and M. S. Gordon, "The construction and interpretation of MCSCF wavefunctions," Annual Review of Physical Chemistry, vol. 49, pp. 233-266, 1998.

[27] A. Pross and S. S. Shaik, "A qualitative valence-bond approach to organic reactivity," Accounts of Chemical Research, vol. 16, p. 363, 1983.

[28] H. E. Zimmerman, "The Möbius-Hückel concept in organic chemistry. Application to organic molecules and reactions," Accounts of Chemical Research, vol. 4, no. 8, pp. 272-280, 1971.

[29] E. Heilbronner, "Hückel molecular orbitals of Mőbius-type conformations of annulenes," Tetrahedron Letters, vol. 5, no. 29, pp. 1923-1928, 1964.

[30] P. W. Fowler and H. S. Rzepa, "Aromaticity rules for cycles with arbitrary numbers of half-twists," Physical Chemistry Chemical Physics, vol. 8, no. 15, pp. 1775-1777, 2006.

[31] S. Zilberg and Y. Haas, "Locating electronic degeneracies of polyatomic molecules: a general method for nonsymmetric molecules," The Journal of Physical Chemistry A, vol. 107, no. 8, pp. 1222-1227, 2003.

[32] S. Zilberg and Y. Haas, "The electron-pair origin of antiaromaticity: spectroscopic manifestations," International Journal of Quantum Chemistry, vol. 71, no. 2, pp. 133-145, 1999. 
[33] L. Salem, “Theory of photochemical reactions," Science, vol. 191, pp. 822-830, 1976.

[34] W. Domcke, H. Koppell, and L. S. Cederbaum, "Spectroscopic effects of comical intersections of molecular-potential energy surfaces," Molecular Physics, vol. 43, no. 4, pp. 851-875, 1981.

[35] M. V. Berry, "Quantal phase factors accompanying adiabatic changes," Proceedings of the Royal Society Series A, vol. 392, no. 1802, pp. 45-57, 1984.

[36] G. J. Atchity, S. S. Xantheas, and K. Ruedenberg, "Ptential energy surfaces near intersections," The Journal of Chemical Physics, vol. 95, pp. 1862-1876, 1990.

[37] Y. Haas, S. Cogan, and S. Zilberg, "The use of elementary reaction coordinates in the search for conical intersections," International Journal of Quantum Chemistry, vol. 102, no. 5, pp. 961-970, 2005.

[38] S. Matsika, "Three-state conical intersections in nucleic acid bases," The Journal of Physical Chemistry A, vol. 109, no. 33, pp. 7538-7545, 2005.

[39] A. J. C. Varandas, "Geometrical phase effect in JahnTeller systems: Twofold electronic degeneracies and beyond," Chemical Physics Letters, vol. 487, no. 1-3, pp. 139-146, 2010.

[40] J. A. Gámez, L. Serrano-andres, and M. Yáñez, “Two- and threestate conical intersections in the electron capture dissociation of disulfides: the importance of multireference calculations," International Journal of Quantum Chemistry, vol. 111, no. 13, pp. 3316-3323, 2011.

[41] P. Celani, F. Bernardi, M. Olivucci, and M. A. Robb, "Excitedstate reaction pathways for s-cis buta-1,3-diene," The Journal of Chemical Physics, vol. 102, no. 14, pp. 5733-5742, 1995.

[42] B. Dick, Y. Haas, and S. Zilberg, "Locating conical intersections relevant to photochemical reactions," Chemical Physics, vol. 347, pp. $65-77,2008$.

[43] M. Ben-Nun and T. D. Martinez, "Photodynamics of ethylene: ab initio studies of conical intersections," Chemical Physics, vol. 259, pp. 237-247, 2000.

[44] Y. Haas and S. Zilberg, "Conical intersections in molecular photochemistry: the phase-change approach," in The Role of Degenerate States in Chemistry, vol. 124 of Advances in Chemical Physics, pp. 433-504, John Wiley \& Sons, New York, NY, USA, 2002.

[45] S. Zilberg and Y. Haas, "The singlet-state photophysics and photochemistry of polyenes: application of the twin-state model and of the phase-change theorem," The Journal of Physical Chemistry A, vol. 103, pp. 2364-2374, 1999.

[46] S. A. Le, J. M. White, and W. A. Noyes, "Some aspects of benzene vapor phase photochemistry," The Journal of Chemical Physics, vol. 65, no. 7, pp. 2805-2811, 1976.

[47] N. J. Turro, C. A. Renner, and T. J. Katz, "Kinetics and thermochemistry of the rearrangement of benzvalene to benzene. An energy sufficient but non-chemiluminescent reaction," Tetrahedron Letters, vol. 46, pp. 4133-4136, 1976.

[48] S. Vanni, M. Garavelli, and M. A. Robb, "A new formulation of the phase change approach in the theory of conical intersections," Chemical Physics, vol. 347, no. 1-3, pp. 46-56, 2008.

[49] J. J. Palmer, I. N. Ragazos, F. Bemardi, M. Olivucci, and M. A. Robb, "An MC-SCF study of the $\mathrm{S}_{1}$ and $\mathrm{S}_{2}$ photochemical reactions of benzene," Journal of the American Chemical Society, vol. 115, no. 2, pp. 673-682, 1993.
[50] H. F. Bettinger, P. R. Schreiner, H. F. Schaefer III, and P. v. R. Schleyer, "Rearrangements on the $\mathrm{C}_{6} \mathrm{H}_{6}$ potential energy surface and the topomerization of benzene," Journal of the American Chemical Society, vol. 120, pp. 5741-5750, 1998.

[51] S. Cogan, A. Kahan, S. Zilberg, and Y. Haas, "Photophysics of (1-Butyl-4-(1H-inden-1-ylidene)-1,4-dihydropyridine (BIDP): an experimental test for conical intersections," The Journal of Physical Chemistry A, vol. 112, pp. 5604-5612, 2008.

[52] S. Cogan and Y. Haas, "Self-sensitized photo-oxidation of paraindenylidene-dihydropyridine derivatives," Journal of Photochemistry and Photobiology A: Chemistry, vol. 193, no. 1, pp. 2532, 2008.

[53] S. Alfalah, O. Deeb, S. Zilberg, and Y. Haas, "Solvent effect on the conical intersection of 4-cyclopentadienylidene-1,4dihydropyridine (CPDHP)," Chemical Physics Letters, vol. 459, no. 1-6, pp. 100-104, 2008.

[54] A. Kahan, A. Wand, S. Ruhman, S. Zilberg, and Y. Haas, "Solvent tuning of a conical intersection: direct experimental verification of a theoretical prediction," The Journal of Physical Chemistry A, vol. 115, no. 40, pp. 10854-10861, 2011.

[55] J.-M. L. Pecourt, J. Peon, and B. Kohler, "Ultrafast internal conversion of electronically excited RNA and DNA nucleosides in water," Journal of the American Chemical Society, vol. 122, no. 38, pp. 9348-9349, 2000.

[56] P. C. Ke, C. Naumann, and P. Dubin, "New developments in polymer analytics II. Advances in polymer science," Journal of the American Chemical Society, vol. 123, pp. 5166-5166, 2001.

[57] J.-M. L. Pecourt, J. Peon, and B. Kohler, "DNA excited-state dynamics: ultrafast internal conversion and vibrational cooling in a series of nucleosides," Journal of the American Chemical Society, vol. 123, no. 42, pp. 10370-10378, 2001.

[58] N. Ismail, L. Blancafort, M. Olivucci, B. Kohler, and M. Robb, "Ultrafast decay of electronically excited singlet cytosine via a $\pi, \pi^{*}$ to $\mathrm{n}_{\mathrm{O}}, \pi^{*}$ state switch," Journal of the American Chemical Society, vol. 124, pp. 6818-6819, 2002.

[59] D. Tuna, A. L. Sobolewski, and W. Domcke, "Mechanisms of ultrafast excited-state deactivation in adenosine," The Journal of Physical Chemistry A, vol. 118, no. 1, pp. 122-127, 2014.

[60] L. Blancafort and M. A. Robb, "Key role of a threefold state crossing in the ultrafast decay of electronically excited cytosine," The Journal of Physical Chemistry A, vol. 108, pp. 10609-10614, 2004.

[61] G. Steinhauser and T. M. Klapötke, “'“Green” pyrotechnics: a chemist's challenge," Angewandte Chemie International Edition, vol. 47 , no. 18 , pp. 3330-3347, 2008.

[62] J. J. Sabatini, A. V. Nagori, G. Chen, P. Chu, R. Damavarapu, and T. M. Klapötke, "High-nitrogen-based pyrotechnics: longerand brighter-burning, perchlorate-free, red-light illuminants for military and civilian applications," Chemistry, vol. 18, no. 2, pp. 628-631, 2012.

[63] A. Vij, J. G. Pavlovich, W. W. Wilson, V. Vij, and K. O. Christe, "Experimental detection of the pentaazacyclopentadienide (pentazolate) anion, cyclo- $\mathrm{N}_{5}$," Angewandte Chemie International Edition, vol. 41, pp. 3051-3054, 2002.

[64] H. Östmark, S. Wallin, T. Brinck et al., "Detection of pentazolate anion $\left(\right.$ cyclo- $\mathrm{N}_{5}^{-}$) from laser ionization and decomposition of solid p-dimethylaminophenylpentazole," Chemical Physics Letters, vol. 379, no. 5-6, pp. 539-546, 2003. 
[65] L. Belau, Y. Haas, and S. Zilberg, "Formation of the cyclopentazolate $\mathrm{N}_{5}{ }^{-}$anion by high-energy dissociation of phenylpentazole anions," The Journal of Physical Chemistry A, vol. 108, no. 52, pp. 11715-11720, 2004.

[66] A. Cembran, F. Bernardi, M. Garavelli, L. Gagliardi, and G. Orlandi, "On the mechanism of the cis-trans isomerization in the lowest electronic states of azobenzene: S-0, S-1, and T-1," Journal of the American Chemical Society, vol. 126, pp. 32343243, 2004.

[67] W. R. Browne and B. L. Feringa, "Light switching of molecules on surfaces," Annual Review of Physical Chemistry, vol. 60, pp. 407-428, 2009.

[68] B. C. Arruda and R. J. Sension, "Ultrafast polyene dynamics: the ring opening of 1,3-cyclohexadiene derivatives," Physical Chemistry Chemical Physics, vol. 16, pp. 4439-4455, 2014.

[69] T. Cusati, G. Granucci, E. Martínez -Núñez, F. Martini, M. Persico, and S. Vázquez, "Semiempirical hamiltonian for simulation of azobenzene photochemistry," The Journal of Physical Chemistry A, vol. 116, no. 1, pp. 98-110, 2012.

[70] G. Tiberio, L. Muccioli, R. Berardi, and C. Zannoni, "How does the trans-cis photoisomerization of azobenzene take place in organic solvents?” ChemPhysChem, vol. 11, pp. 1018-1028, 2010.

[71] J. J. Blavins, D. L. Cooper, and P. B. Karadakov, "Modern valence bond description of the electronic mechanisms of $\mathrm{S}_{N} 2$ identity reactions," The Journal of Physical Chemistry, vol. 108, pp. 914920, 2004.

[72] S. Shaik and P. C. Hiberty, "Myth and reality in the attitude toward valence-bond (VB) theory: are its "Failures" real?" Helvetica Chimica Acta, vol. 86, no. 4, pp. 1063-1084, 2003.

[73] G. A. Worth and L. S. Cederbaum, "Mediation of ultrafast electron transfer in biological systems by conical intersection," Chemical Physics Letters, vol. 338, no. 4-6, pp. 219-223, 2001.

[74] D. R. Yarkony, "On the role of conical intersections of two potential energy surfaces of the same symmetry in photodissociation. I. $\mathrm{CH}_{3} \mathrm{SH} \rightarrow \mathrm{CH}_{3} \mathrm{~S}+\mathrm{H}$ and $\mathrm{CH}_{3}+\mathrm{SH}$,' The Journal of Chemical Physics, vol. 100, no. 5, pp. 3639-3644, 1994. 

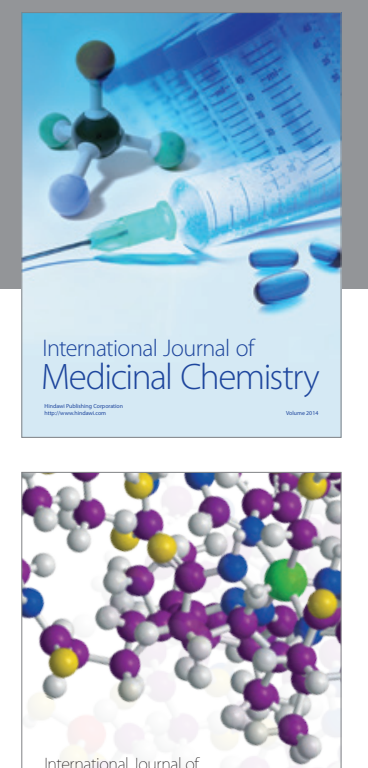

\section{Carbohydrate} Chemistry

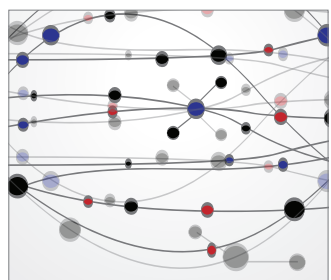

The Scientific World Journal
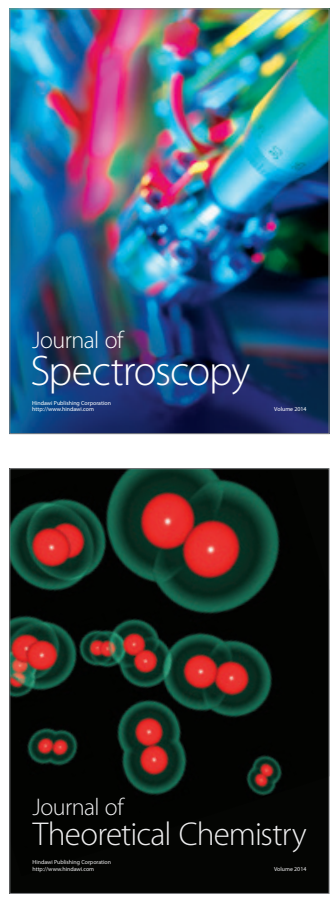
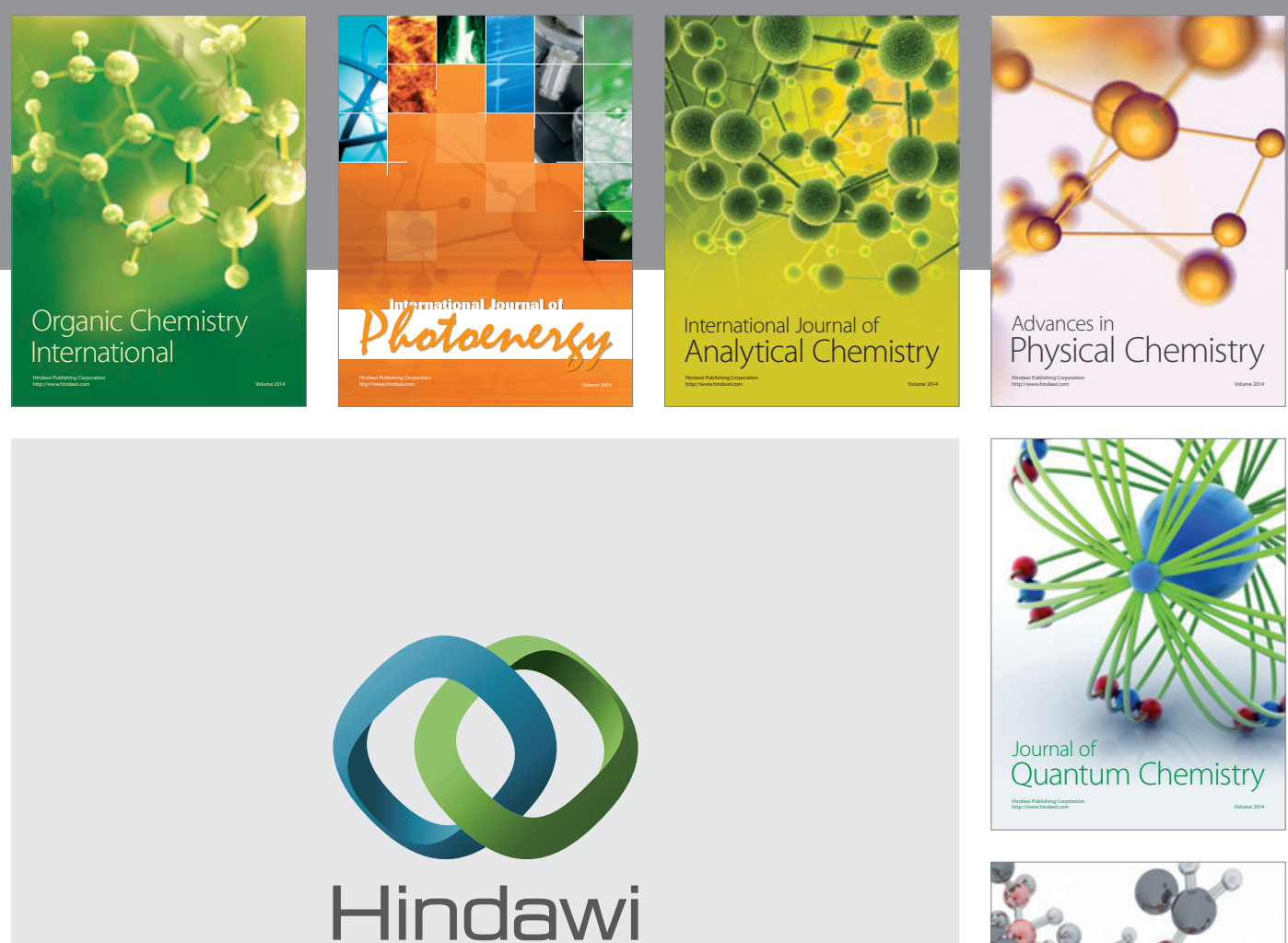

Submit your manuscripts at

http://www.hindawi.com

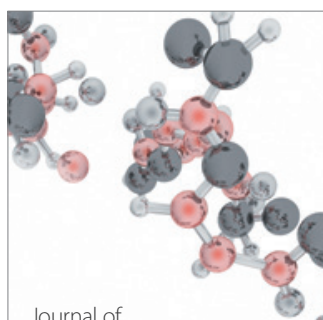

Analytical Methods

in Chemistry

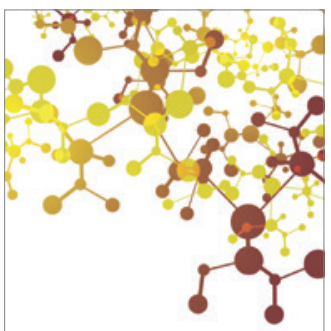

Journal of

Applied Chemistry

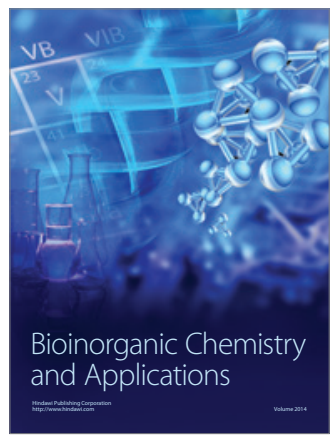

Inorganic Chemistry
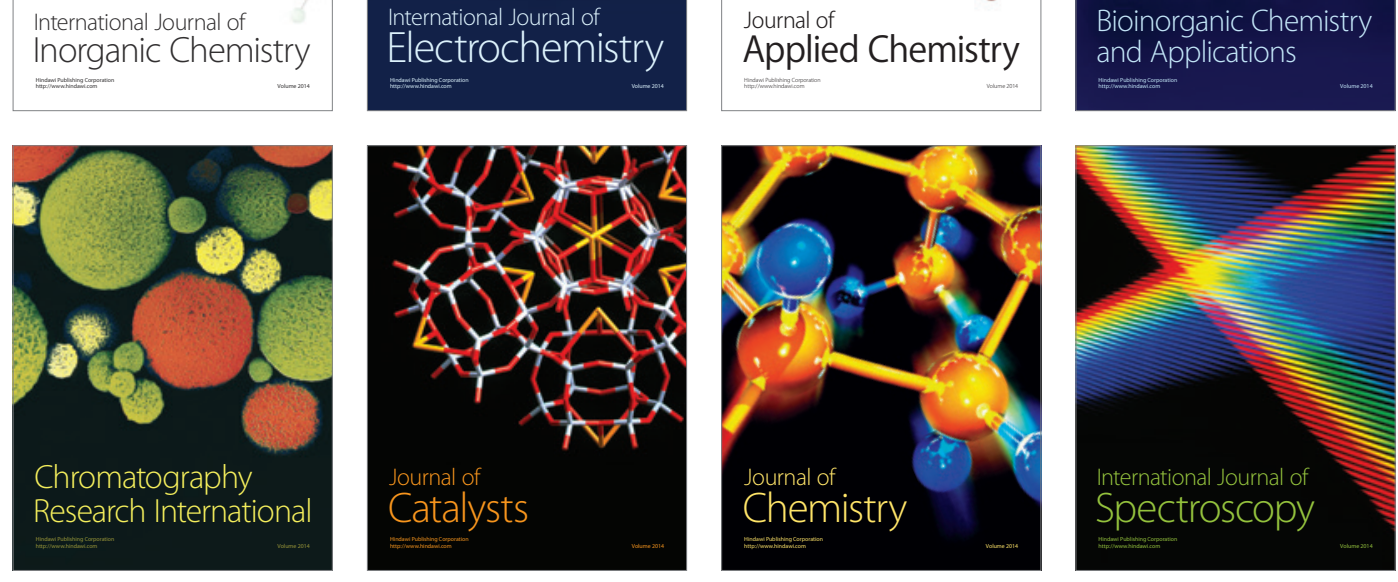